\title{
Utilizing CT with Maximum Intensity Projection Reconstruction Bypassing CTA Improves Time to Groin Puncture in Large Vessel Occlusion Stroke Thrombectomy
}

\author{
Kunakorn Atchaneeyasakul $^{\mathrm{a}}$ Anita Tipirneni ${ }^{\mathrm{a}}$ Priyank Khandelwal ${ }^{\mathrm{a}}$ \\ Vasu Saini $^{a} \quad$ Richard Ronca $^{a}$ Steven Lord ${ }^{a}$ Samir Sur ${ }^{b}$ Luis Guada ${ }^{a}$ \\ Kevin Ramdas $^{a}$ Eric Peterson ${ }^{b}$ Dileep Yavagal ${ }^{a}$ b \\ Departments of a Neurology and ${ }^{b}$ Neurological Surgery, University of Miami Miller School of \\ Medicine, Miami, FL, USA
}

\section{Keywords}

Stroke $\cdot$ Maximum intensity projection $\cdot$ Thrombectomy $\cdot$ CT angiography

\begin{abstract}
Background and Purpose: Prior to thrombectomy for proximal anterior circulation large vessel occlusion (LVO) stroke, recent trials have utilized CT angiography (CTA) for vascular imaging immediately following noncontrast CT (NCCT) for decision-making, but thin-section NCCT with automated maximum intensity projection (MIP) reconstruction also has high accuracy in demonstrating the site of an occluding thrombus. We hypothesized that performing thinsection NCCT with MIP alone prior to thrombectomy improves the time to groin puncture (GP) compared to performing CTA after NCCT. Materials and Methods: We performed a retrospective cohort study of anterior circulation LVO thrombectomy at our tertiary care academic medical center. All stroke patients evaluated with thin-section NCCT $(0.625 \mathrm{~mm})$ with automated MIP reconstructions alone and those who had additional CTA were included. We excluded transfer patients, in-hospital strokes, posterior circulation strokes, and patients that were evaluated with stroke imaging other than NCCT or CTA prior to thrombectomy. The study groups were compared for duration from NCCT to GP and total stroke imaging duration. $\boldsymbol{R e}$ sults: From March 2008 through August 2015, 34 thrombectomy patients met the inclusion/ exclusion criteria - 13 in the NCCT and 20 in the NCCT+CTA group. The total stroke imaging duration was shorter in the NCCT group than in the NCCT+CTA group (2 min [1-6] vs. 28 min [23-65]; $p<0.001$ ). The NCCT-only group had a shorter time from NCCT to GP (68 min [32-99] vs. $104 \min$ [79-128]; $p=0.030$ ). Conclusion: Avoiding advanced imaging for patients with anterior circulation LVO in whom thin-section NCCT with MIPs reveals a hyperdense sign significantly shortens the imaging-to-GP time.




\section{Introduction}

Stroke is one of the leading causes of morbidity and mortality in the USA [1]. Recent studies have shown that acute ischemic stroke patients with anterior circulation large vessel occlusion (LVO) undergoing mechanical thrombectomy have significant improvement in functional outcome compared to those treated with tissue plasminogen activator (tPA) alone [2]. The time to endovascular recanalization also has a significant effect on clinical outcome [3]. These studies utilized CT angiography (CTA) and/or other stroke imaging modalities immediately following noncontrast CT (NCCT) to confirm LVO [4, 5]. However, multiple imaging modalities may delay the time to groin puncture (GP) compared to NCCT alone. Many institutions, including our tertiary academic medical center, utilize CTA to confirm LVO in the stroke care protocol. But CTA is not often performed immediately after NCCT. There are many factors prior to CTA acquisition - including large bore intravenous line insertion - that can potentially delay advanced imaging and, ultimately, the time to GP. For patients with difficult intravenous access, an experienced personnel with an ultrasound guide may require up to $10 \mathrm{~min}$ or more to achieve intravenous access [6]. The maximum intensity projections (MIPs) of thin-slice NCCT further improve the sensitivity and accuracy of identifying acute thrombi of LVO in the anterior circulation by detecting "hyperdense signs" reliably, and CTA confirmation may not be necessary in selected cases [7]. We hypothesized that thin-section NCCT alone improves the time to GP when compared to CTA after NCCT.

\section{Methods}

We performed a retrospective cohort study of a prospectively collected database of ischemic stroke patients receiving mechanical thrombectomy at our tertiary care academic medical center. This study received approval from our institutional review board. The inclusion criteria covered patients with any anterior circulation occlusion receiving mechanical thrombectomy of any type and NCCT with thin sections (0.625-mm slices) and software-automated MIP. Figure 1 shows a thrombus seen on CTA compared to NCCT with MIP reconstruction. The exclusion criteria filtered out patients transferred from other facilities, in-hospital stroke patients, posterior circulation stroke patients, or those having undergone stroke imaging other than NCCT or CTA prior to thrombectomy.

We evaluated the patient demographics including age at diagnosis, gender, medical comorbidities (hypertension, diabetes mellitus, hyperlipidemia, and atrial fibrillation), and smoking history within the past 2 years.

The patients were divided into 2 groups based on the stroke imaging results obtained prior to thrombectomy, i.e., an NCCT-only group and an NCCT+CTA group. Per our institutional protocol, patients were immediately transported to NCCT on ER arrival. All stroke NCCTs included were thin-section NCCTs (0.625-mm slices) with software-automated MIP reconstructions available within 2 min of CT completion. The neuroradiologist on call evaluated the stroke NCCT and CTA images. When "hyperdense signs" were seen on the thin-section MIPs, the stroke team could forgo CTA and bring the patient directly to angiography in order to prevent treatment delay. A stroke care team initiated intravenous access and blood draw at the CT suite immediately after completion of the NCCT. In cases meeting the criteria for intravenous thrombolysis, tPA was given immediately after NCCT.

We evaluated the time periods from last known well to NCCT, from NCCT to GP, the total imaging duration prior to GP, and from last known well to GP. The percentage of patients treated with IV tPA and with a stent retriever was evaluated. The clinical outcomes assessed included admission/discharge National Institutes of Health Stroke Scale (NIHSS) score, discharge modified Rankin Scale (mRS) score, and reperfusion with TICI $2 \mathrm{~b} / 3$ flow.

Statistical analysis was performed using the Statistical Package for the Social Sciences (SPSS version 22; IBM Corporation, Armonk, NY, USA). Group comparisons were made with the $t$ test and the $\chi^{2}$ test as appropriate. Statistical significance was determined as a $p$ value $<0.05$. 


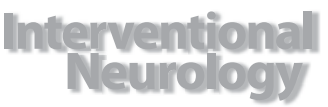

\begin{tabular}{l|l}
\hline Intervent Neurol 2017;6:147-152 \\
\hline DOI: 10.1159/000464300 & $\begin{array}{l}\text { @ 2017 S. Karger AG, Basel } \\
\text { www.karger.com/ine }\end{array}$ \\
\hline
\end{tabular}

Atchaneeyasakul et al.: Utilizing CT with MIP Reconstruction Bypassing CTA Improves Time to Groin Puncture in LVO Stroke Thrombectomy
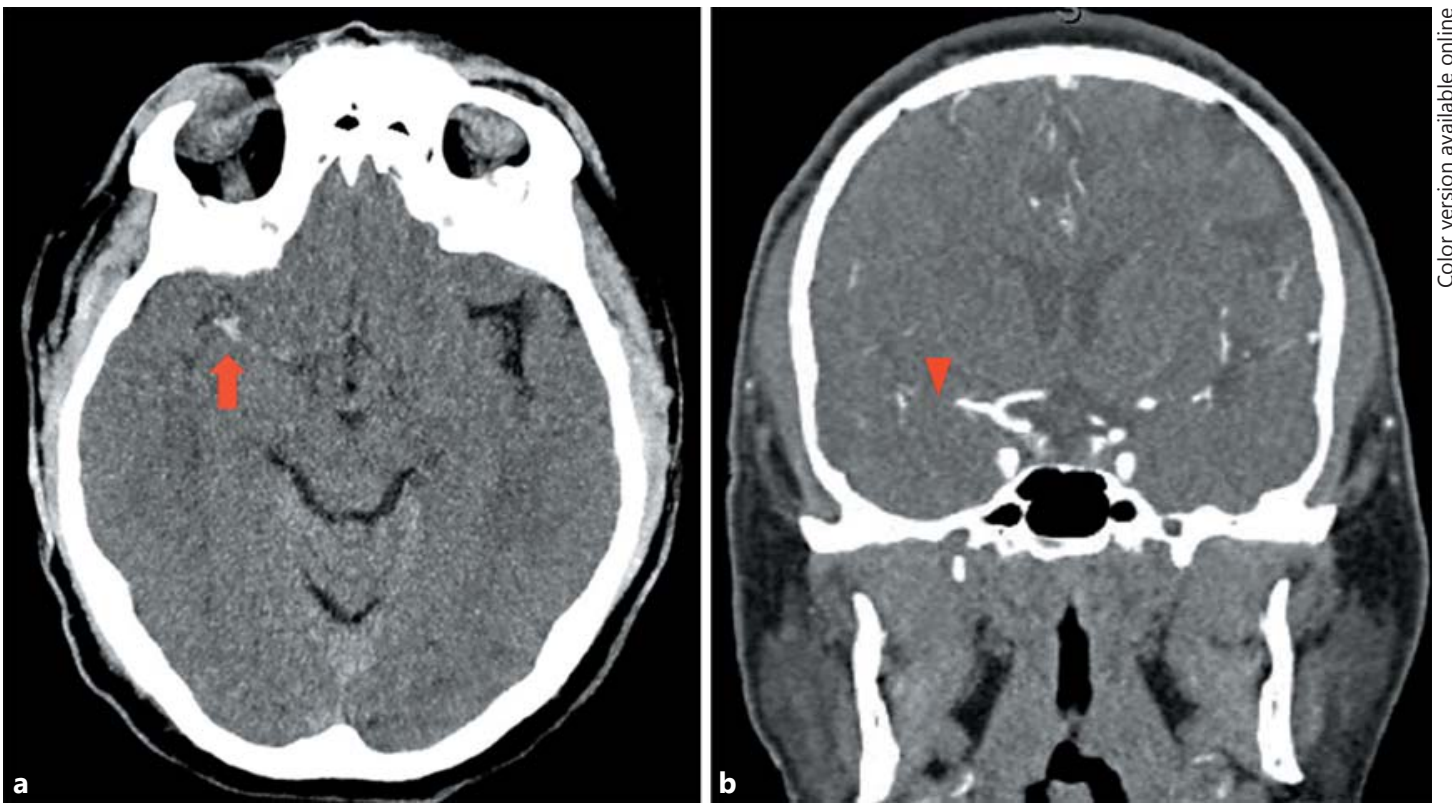

Fig. 1. a A thrombus (arrow) is seen on noncontrast CT with maximum intensity projection reconstruction of right middle cerebral artery (MCA) M1 occlusion. b CT angiography with contrast, confirming the right MCA M1 cutoff at the same location (arrowhead) as the thrombus seen on noncontrast CT with maximum intensity projection reconstruction.

\section{Results}

From March 2008 through August 2015, 34 thrombectomy patients met the inclusion criteria: 14 in the NCCT group and 20 in the NCCT+CTA group.

Univariate analysis of the demographics, medical history, occlusion site, IV tPA usage, stent retriever usage, and clinical outcomes is shown in Table 1. M1 segment occlusion was noted in $53.8 \%$ of the NCCT group and in 55\% of the NCCT+CTA group. A "hyperdense sign" was accurately detected in $85 \%$ of the NCCT+CTA group. IV tPA was given to $100 \%$ of the NCCT group and $55 \%$ of the NCCT+CTA group. There was no significant difference in usage of a stent retriever or successful reperfusion.

The time delay comparison between the 2 groups is shown in Table 2 . We found a significantly shorter delay for NCCT to GP (median $68 \mathrm{~min}$ [32-99] vs. $104 \mathrm{~min}$ [79-128]; $p=$ 0.030 ). There also was a significantly shorter total stroke imaging duration in the NCCT group (median $2 \min [1-6]$ vs. $28 \min$ [23-65]; $p<0.001$ ). There was no significant difference between the groups in delay from last known well to imaging initiation. There was also no significant difference in duration from GP to thrombectomy device deployment between the 2 groups.

Regarding clinical outcomes, there was no significant different in mRS and NIHSS scores on discharge, as shown in Table 1. 
Atchaneeyasakul et al.: Utilizing CT with MIP Reconstruction Bypassing CTA Improves Time to Groin Puncture in LVO Stroke Thrombectomy

Table 1. Baseline characteristics, clinical outcomes, and interventions received of the subjects in the NCCT and NCCT+CTA groups

\begin{tabular}{lccc}
\hline & $\begin{array}{l}\text { NCCT } \\
(n=13)\end{array}$ & $\begin{array}{l}\text { NCCT+CTA } \\
(n=20)\end{array}$ & $p$ value \\
\hline Mean age \pm SD, years & $63.8 \pm 18.3$ & $71.2 \pm 15.5$ & 0.23 \\
Female gender, $n(\%)$ & $7(53.8)$ & $10(50)$ & 0.83 \\
Hypertension, $n(\%)$ & $8(61.5)$ & $14(70)$ & 0.61 \\
Diabetes mellitus, $n(\%)$ & $4(30.8)$ & $3(15)$ & 0.28 \\
Hyperlipidemia, $n(\%)$ & $4(30.8)$ & $4(20)$ & 0.48 \\
Atrial fibrillation, $n(\%)$ & $4(30.8)$ & $3(15)$ & 0.28 \\
Cigarette smoking, $n(\%)$ & $2(15.4)$ & $7(35)$ & 0.22 \\
Occlusion site, $n(\%)$ & & & 0.77 \\
$\quad$ MCA M1 & $7(53.8)$ & $11(55)$ & \\
MCA M2 & $5(38.5)$ & $6(30)$ & $3(15)$ \\
$\quad 1 C A$ & $1(7.7)$ & $0(0)$ & 0.68 \\
Other & $0(0)$ & $15.7 \pm 6.2$ & 0.74 \\
Mean NIHSS score \pm SD at presentation & $16.6 \pm 6.2$ & $22.7 \pm 18.6$ & 0.33 \\
Mean mRS score \pm SD at discharge & $20.5 \pm 17.7$ & $4.1 \pm 2.2$ & $0.003^{*}$ \\
Received IV tPA, $n(\%)$ & $3.8 \pm 2.2$ & $11(55)$ & 0.24 \\
Stent retriever usage, $n$ (\%) & $14(100)$ & $18(90)$ & 0.25 \\
Successful reperfusion, $n$ (\%) & $13(100)$ & $16(80)$ & \\
\hline
\end{tabular}

NCCT, noncontrast CT; CTA, CT angiography; MCA, middle cerebral artery; mRS, modified Rankin Scale; tPA, tissue plasminogen activator. * Significant $p$ value.

Table 2. Comparison of durations ( $\mathrm{min}$ ) between the NCCT and the NCCT+CTA group prior to thrombectomy

\begin{tabular}{lccc}
\hline & $\begin{array}{l}\text { NCCT } \\
(n=14)\end{array}$ & $\begin{array}{l}\text { NCCT+CTA } \\
(n=20)\end{array}$ & $p$ value \\
& $68(32-99)$ & $104(79-128)$ & $0.030^{*}$ \\
Duration from NCCT to GP & $2(1-6)$ & $28(23-65)$ & $<0.001 *$ \\
Total imaging duration & $97(59-144)$ & $96(58-239)$ & 0.677 \\
Duration from last known well to NCCT & $170(130-214)$ & $207(156-324)$ & 0.108 \\
Duration from last known well to GP & $22.5(15.5-36.75)$ & $20.5(16-39.25)$ & 0.966 \\
Duration from GP to thrombectomy device deployment & & \\
\hline
\end{tabular}

Values are presented as median (IQR 25th-75th percentile). NCCT, noncontrast CT; CTA, CT angiography; GP, groin puncture. * Significant $p$ value.

\section{Discussion}

In our study, we found that avoiding advanced vascular imaging (CTA) in patients in whom NCCT with MIP images demonstrates the site of occlusion significantly shortens the door-to-puncture time. This improvement in time was approximately $30 \mathrm{~min}$ in our patient cohort. Shortening the time to reperfusion is associated with improved functional outcome, and the time span could be improved by optimization of the time delay prior to GP [3]. Performing CTA after NCCT prior to thrombectomy to confirm LVO is widely done in many institutions, based on the protocol used in a recently published large clinical trial for anterior circulation stroke thrombectomy [8]. In these studies, CTA is performed immediately, without 
Atchaneeyasakul et al.: Utilizing CT with MIP Reconstruction Bypassing CTA Improves Time to Groin Puncture in LVO Stroke Thrombectomy

interruption, after NCCT, in most cases minimizing the delay. However, in the setting of many stroke centers - including our institution, which is a large referral center for mechanical thrombectomy - in selected cases performing CTA after NCCT may potentially be delayed by many factors including difficult intravenous access or the requirement of stroke team decision-making prior to obtaining CTA. These factors may have contributed to the delay seen in our study.

Thin-slice NCCT with MIP reconstruction is a technique of NCCT imaging that shows a higher probability of detecting LVO than does routine 5-mm-slice NCCT, and it may allow one to forgo CTA to shorten delays to GP [7]. In our study, the "hyperdense sign" in thin-slice MIP reconstructions was observed in $85.3 \%$ of the cases. In the patients excluded from our analysis, standard 5-mm-slice NCCT showed a $28.6 \%$ detection rate. In our NCCT+CTA group, 17 patients (85\%) had a hyperdense cerebral vessel detected. This implies that most patients could have bypassed CTA if needed and gone directly to angiography for thrombectomy, minimizing the delay, especially in the case of an inevitable delay due to difficulty in gaining intravenous access.

CTA has many advantages over NCCT, including a higher sensitivity for detecting LVO, visualization of carotid vessels, and the tortuosity of the aortic arch as part of stroke workup and thrombectomy planning, especially for choosing the appropriate approach to vascular access in the case of a difficult vascular anatomy (including femoral artery or radial artery access) [9]. However, in the hyperacute period of LVO stroke, the time to GP should be minimized.

Given our findings, we propose that all NCCT scans should be performed with MIP reconstruction. The treating physician may forgo CTA in the setting of a positive "hyperdense sign" on NCCT with MIP reconstruction, especially in the setting of an inevitable delay in obtaining CTA. Performing MIP reconstruction is a default and automated process for all acute stroke cases at our institution. No extra step is required once the acute stroke protocol CT has been chosen, and conventional NCCT images and MIPs become available less than 2 min after scanning. In the absence of a hyperdense sign on NCCT with MIP reconstruction, CTA should be performed without delay. This protocol could in selected cases reduce an unnecessary delay when there already is a foreseeable delay to obtaining CTA.

Although a significant reduction in time was seen in the NCCT-only group, there was no significant difference in mRS or NIHSS scores on discharge. However, our study samples were small and likely not powered to see significant differences. Larger samples are needed to further assess clinical outcomes.

The limitations of our study include its retrospective nature, the small sample size, and usage of a single-institution database which may not be generalizable. The small sample size was due to low rates of thrombectomy without CTA and the exclusion criteria of transfer/ in-house strokes. A larger prospective trial is needed to further confirm our findings.

\section{Conclusion}

Avoiding advanced imaging in patients with anterior circulation LVO in whom thinsection NCCT with MIPs reveals a hyperdense sign significantly shortens the imaging-to-GP time.

\section{Disclosure Statement}

The authors have no conflicts of interest to disclose. 
Atchaneeyasakul et al.: Utilizing CT with MIP Reconstruction Bypassing CTA Improves Time to Groin Puncture in LVO Stroke Thrombectomy

\section{References}

1 Towfighi A, Saver JL: Stroke declines from third to fourth leading cause of death in the United States: historical perspective and challenges ahead. Stroke 2011;42:2351-2355.

2 Goyal M, Menon BK, van Zwam WH, Dippel DW, Mitchell PJ, Demchuk AM, Dávalos A, Majoie CB, van der Lugt A, de Miquel MA, Donnan GA, Roos YB, Bonafe A, Jahan R, Diener HC, van den Berg LA, Levy EI, Berkhemer OA, Pereira VM, Rempel, Millán M, Davis SM, Roy D, Thornton J, Román LS, Ribó M, Beumer D, Stouch B, Brown S, Campbell BC, van Oostenbrugge RJ, Saver JL, Hill MD, Jovin TG; HERMES Collaborators: Endovascular thrombectomy after large-vessel ischaemic stroke: a meta-analysis of individual patient data from five randomised trials. Lancet 2016;387:1723-1731.

3 Nogueira RG, Smith WS, Sung G, Duckwiler G, Walker G, Roberts R, Saver JL, Liebeskind DS; MERCI and Multi MERCI Writing Committee: Effect of time to reperfusion on clinical outcome of anterior circulation strokes treated with thrombectomy: pooled analysis of the MERCI and Multi MERCI trials. Stroke 2011;42:31443149.

4 Saver JL, Goyal M, Bonafe A, Diener HC, Levy EI, Pereira VM, Albers GW, Cognard C, Cohen DJ, Hacke W, Jansen O, Jovin TG, Mattle HP, Nogueira RG, Siddiqui AH, Yavagal DR, Devlin TG, Lopes DK, Reddy V, du Mesnil de Rochemont R, Jahan R; SWIFT PRIME Investigators: Solitaire ${ }^{\mathrm{TM}}$ with the Intention for Thrombectomy as Primary Endovascular Treatment for Acute Ischemic Stroke (SWIFT PRIME) trial: protocol for a randomized, controlled, multicenter study comparing the Solitaire revascularization device with IV IPA with IV tPA alone in acute ischemic stroke. Int J Stroke 2015;10:439-448.

5 Campbell BC, Mitchell PJ, Kleinig TJ, Dewey HM, Churilov L, Yassi N, Yan B, Dowling RJ, Parsons MW, Oxley TJ, Wu TY, Brooks M, Simpson MA, Miteff F, Levi CR, Krause M, Harrington TJ, Faulder KC, Steinfort BS, Priglinger M, Ang T, Scroop R, Barber PA, McGuinness B, Wijeratne T, Phan TG, Chong W, Chandra RV, Bladin CF, Badve M, Rice H, de Villiers L, Ma H, Desmond PM, Donnan GA, Davis SM; EXTEND-IA Investigators: Endovascular therapy for ischemic stroke with perfusion-imaging selection. N Engl J Med 2015;372:1009-1018.

6 Scoppettuolo G, Pittiruti M, Pitoni S, Dolcetti L, Emoli A, Mitidieri A, Migliorini I, Annetta MG: Ultrasoundguided "short" midline catheters for difficult venous access in the emergency department: a retrospective analysis. Int J Emerg Med 2016;9:3.

7 Riedel CH, Zoubie J, Ulmer S, Gierthmuehlen J, Jansen O: Thin-slice reconstructions of nonenhanced CT images allow for detection of thrombus in acute stroke. Stroke 2012;43:2319-2323.

8 Saver JL, Goyal M, Bonafe A, Diener HC, Levy EI, Pereira VM, Albers GW, Cognard C, Cohen DJ, Hacke W, Jansen O, Jovin TG, Mattle HP, Nogueira RG, Siddiqui AH, Yavagal DR, Baxter BW, Devlin TG, Lopes DK, Reddy VK, du Mesnil de Rochemont R, Singer OC, Jahan R; SWIFT PRIME Investigators: Stent-retriever thrombectomy after intravenous t-PA vs t-PA alone in stroke. N Engl J Med 2015;372:2285-2295.

9 Lev MH, Farkas J, Rodriguez VR, Schwamm LH, Hunter GJ, Putman CM, Rordorf GA, Buonanno FS, Budzik R, Koroshetz WJ, Gonzalez RG: CT angiography in the rapid triage of patients with hyperacute stroke to intraarterial thrombolysis: accuracy in the detection of large vessel thrombus. J Comput Assist Tomogr 2001;25: 520-528. 